\title{
Change in Antidepressant Use After Initiation of ADHD Medication in Japanese Adults with Comorbid Depression: A Real-World Database Analysis
}

\author{
Chika Sakai ${ }^{\prime}$ \\ Toshinaga Tsuji $\mathbb{D}^{\prime}$ \\ Toru Nakai' \\ Yuki Namba' \\ Hirokazu Mishima' \\ Masakazu Fujiwara ${ }^{2}$ \\ Hisato Matsunaga ${ }^{3}$ \\ 'Medical Affairs Department, Shionogi \& \\ Co. Ltd, Osaka, Japan; ${ }^{2}$ Data Science \\ Office, Shionogi \& Co. Ltd, Osaka, Japan; \\ ${ }^{3}$ Department of Neuropsychiatry, Hyogo \\ College of Medicine, Hyogo, Japan
}

Purpose: To better understand the treatment of comorbid depression in adults with attention-deficit/hyperactivity disorder (ADHD) by investigating the prescription patterns of antidepressants, anxiolytics, and hypnotics after commencing ADHD medication.

Patients and Methods: In this retrospective observational study in Japan, the data of patients initiating ADHD medication while already receiving antidepressants (ADHD group) and of patients prescribed antidepressants but not diagnosed with ADHD (control group) were extracted from an electronic medical record database. Additionally, one-to-one matching for patients in both groups was performed using sex, age, baseline dosage of antidepressants, and any comorbid psychiatric disorders as covariates. The observation period included a 1-month baseline period and a 6-month follow-up period. The percentage of patients prescribed antidepressants and the mean prescribed dosages were compared between matched-cohort groups. Prescriptions for anxiolytics and hypnotics were also assessed.

Results: In the matched cohorts, consisting of 239 patients in the ADHD group and 239 patients from the unmatched control cohort of 10,485, the percentage of patients prescribed antidepressants decreased from baseline in both groups to $94.1 \%$ in the ADHD group and $89.5 \%$ in the control group during the first month of follow-up, and $77.0 \%$ and $78.7 \%$, respectively, during the last month. There were no significant differences between groups in the percentages of patients prescribed antidepressants or in the mean prescribed dosages of antidepressants at any time point over the follow-up period. Prescribed dosages of anxiolytics and hypnotics tended to be lower in the ADHD group.

Conclusion: The two groups were medicated similarly with respect to their depressive symptoms over 6 months. Our results suggest that in patients with ADHD and comorbid depression, which is more likely to be more severe than in depression without ADHD, depressive symptoms are managed following initiation of add-on ADHD medication, without requiring higher antidepressant dosages than in patients with depression only.

Keywords: attention-deficit/hyperactivity disorder, MDD, antidepressants, anxiolytics, hypnotics

\section{Introduction}

Attention-deficit/hyperactivity disorder (ADHD) is a neurodevelopmental disorder characterized by hyperactivity, impulsivity, and inattention, as defined in the Diagnostic and Statistical Manual of Mental Disorders, Fifth Edition (DSM-5). ${ }^{1}$ Although ADHD was previously considered a pediatric-specific disorder, it is now
Medical Affairs Department, Shionogi \&

Co. Ltd, 3-1-8, Doshomachi, Chuo-ku,

Osaka-shi, Osaka, 54I-0045, Japan

Tel +8I 662097412

Fax +81 662022239

Email toshinaga.tsuji@shionogi.co.jp 
recognized to often persist into adulthood, ${ }^{2-4}$ with the diagnostic criteria for ADHD in adults currently defined in DSM-5. ${ }^{1}$ While it has been previously assumed that ADHD in adults is a neurodevelopmental disorder that stems from childhood-onset ADHD, results from a longterm longitudinal cohort study showed that this is not necessarily true for most cases, and as such, adult ADHD and childhood ADHD should be considered separate disorders. ${ }^{5}$ A survey study reported an estimated ADHD prevalence of $2.9 \%$ and $16.4 \%$ for narrow ADHD and broad ADHD, respectively, among adults. ${ }^{6}$ The symptoms of ADHD in adults generally involve less hyperactivity than in childhood but may be characterized by inattention and impulsivity. ${ }^{1}$ Overall, adults with ADHD may have persistent difficulties in daily living and employment due to these symptoms, with negative effects on patients' mental health.

ADHD in adults is often associated with comorbid psychiatric disorders, including mood and anxiety disorders. As symptoms of ADHD and comorbid psychopathologies often overlap, the diagnosis and management of comorbid ADHD is often more challenging. ${ }^{7}$ A $22.1 \%$ prevalence of ADHD symptoms in patients with current major depressive disorder (MDD) and a $29.0 \%$ prevalence in patients with comorbid anxiety disorders has been reported. ${ }^{8}$ In ADHD patients, sleep disturbances may lead to significant functional impairments that affect these patients' mood, attention, behavior, school/work performance, and overall quality of life. ${ }^{9}$ A systematic literature review of ADHD and comorbid somatic diseases found associations between ADHD and sleep disorders, with $80 \%$ of adults with ADHD reporting sleep problems. ${ }^{10}$ These comorbid psychiatric disorders often result in delayed diagnosis and treatment of the underlying $\mathrm{ADHD},{ }^{7}$ and, as a result, ADHD symptoms tend to become treatment-refractory and persistent.

Among the previous studies in which depressive symptoms in patients with ADHD were investigated, the effect of a 12-week treatment with methylphenidate hydrochloride extended-release tablets, a common ADHD medication, on depressive symptoms was evaluated. ${ }^{11}$ The results showed a significant reduction in Hamilton Depression Rating Scale scores, indicating an improvement in depressive symptoms in adults with ADHD as a result of this treatment. ${ }^{11}$

The present study was conducted to investigate whether commencing ADHD medication has an effect on prescription continuation and dosages of preexisting antidepressant, anxiolytic, or hypnotic treatments in patients aged 20-40 years, to better understand the medication status of adults with comorbid depression and ADHD. We were also interested in anxiety and sleep disorders, which are common comorbid psychiatric disorders in patients with ADHD. The 20-40 age range was selected to focus on a population of patients that may be experiencing depressive symptoms as a result of difficulties in daily life. In this age group, underlying ADHD tends to be overlooked and is often only discovered when consulting with a doctor about other, more common, psychiatric conditions such as a depression.

\section{Materials and Methods Study Design}

This was a retrospective observational study using data collected from the Japan Medical Data Survey (JAMDAS) electronic medical record database operated by M3 Inc. (Tokyo, Japan). The data of patients who commenced ADHD medication while being prescribed antidepressants were extracted (ADHD group), along with the data of patients who were prescribed antidepressants with no record of ADHD diagnosis, by assessing disease names used for insurance claims or medication (control group).

Index dates were defined to determine the observation period for each patient: in the ADHD group this was the date when a prescription for an ADHD medication was added to an existing prescription for antidepressants, and in the control group this was 1 month after the date the patient was prescribed antidepressants. The observation period was 7 months in total: a 1-month baseline period before the index date and a 6-month follow-up period after the index date (see Figure 1).

\section{Database Selection}

The JAMDAS is a real-world database that includes clinical practice information on approximately 4.4 million patients from approximately 1700 clinics in Japan. This database includes anonymized data, such as disease statistics, prescribed medications, medical treatment provided, and laboratory data. These data are updated and utilized in a real time manner. We selected this database because it includes data from general practitioners, who are likely to be the first point of medical contact for our population of interest: adult patients experiencing depressive symptoms due to underlying ADHD. 


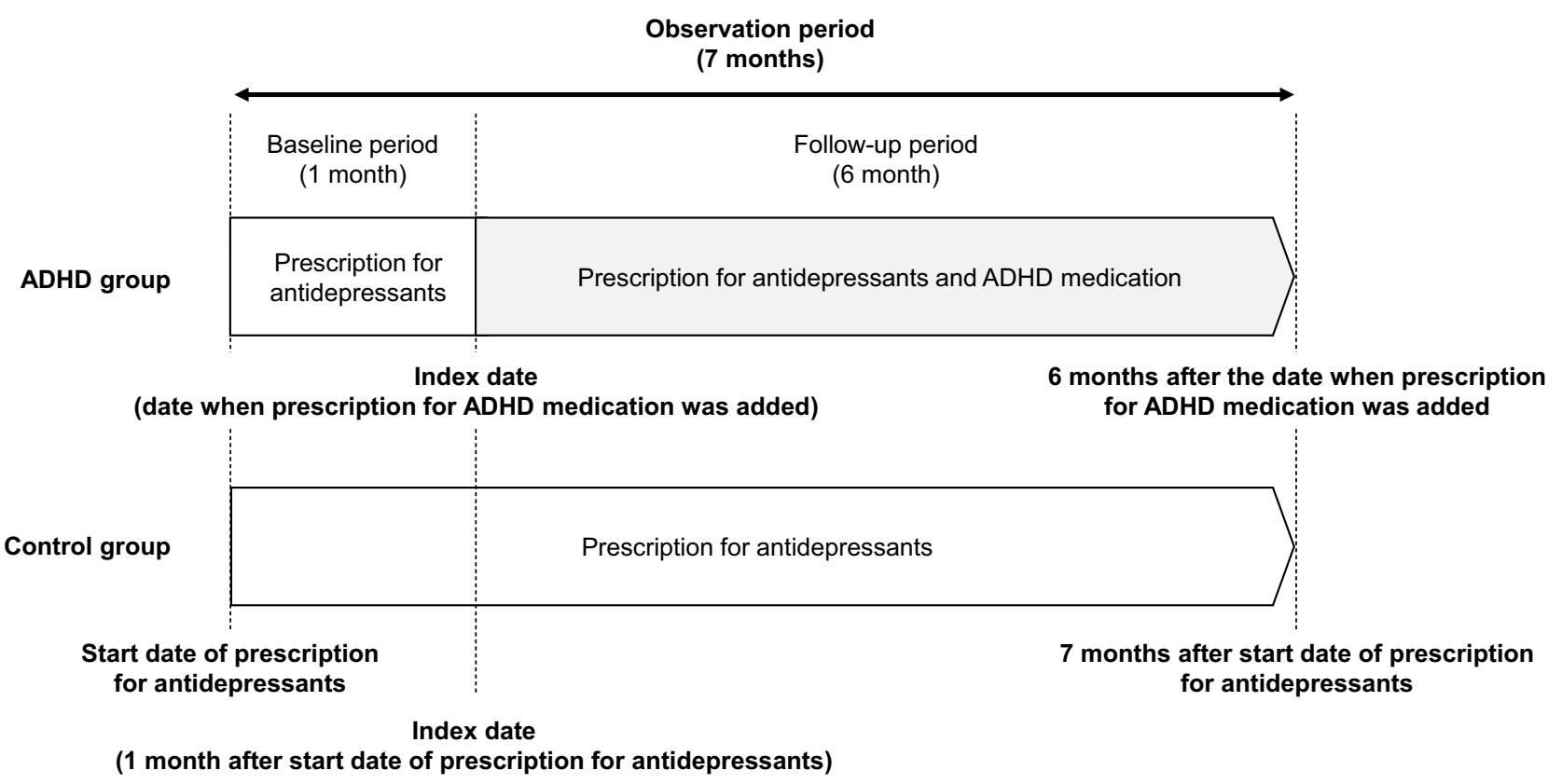

Figure I Study diagram including definition of the evaluation period and index date in each group. Abbreviation: ADHD, attention-deficit/hyperactivity disorder.

\section{Data Extraction}

The inclusion criteria for the ADHD group were: men and women aged 20-40 years; who were prescribed both ADHD medication and antidepressants; who had received at least a 28-day prescription for ADHD medication within the first 30 days of the follow-up period; who had received at least a 14-day prescription for antidepressants during the baseline period; and who were recorded in the JAMDAS database for at least 6 months after the index date.

The inclusion criteria for the control group were: men and women aged 20-40 years; with no record of an ADHD diagnosis and no prescription record for ADHD medication(s); who had received at least a 14-day prescription for antidepressants during the baseline period; and who were recorded in the JAMDAS database for the entire 7 -month observation period. The observation period was set at 7 months because insufficient patient data were available from the JAMDAS database after 7 months. The following data were extracted: disease names or insurance claims, and prescription data including drug name, dosage per day, prescription date, and number of days of prescription.

\section{Endpoints}

The primary endpoint was the percentage of patients still receiving prescriptions for antidepressants at the end of the observational period. The secondary endpoints were the mean prescribed dosages of antidepressants per patient per month (in imipramine [IMP] equivalents), along with the percentages of patients receiving prescriptions for anxiolytics or hypnotics and their mean prescribed dosages per patient per month in diazepam [DZP] equivalents. Only drugs with conversion values to IMP or $\mathrm{DZP}^{12}$ were included in the analyses of mean prescribed dosage. Prescriptions for the hypnotics suvorexant, an orexin receptor antagonist, and ramelteon, a melatonin receptor agonist, were also assessed.

\section{Data Analysis}

After extraction of the data from the database, one-to-one matching of patients in both groups was performed. Propensity scores were estimated using logistic regression models, with patient background factors that may affect antidepressant use as covariates. Patients in the ADHD group were matched to patients in the control group with minimum differences in propensity scores using a caliper of 0.2. Of the patients prescribed both ADHD medication and antidepressants, the same number of propensity-score matched patients from the total number of patients who were prescribed antidepressants was extracted. The patients' background factors at the index date were defined as covariates. The covariates were sex, age, dosage of antidepressants, and the recorded name of any psychiatric 
disorders including bipolar disorder, sleep disorder, and anxiety disorder.

Patient demographics and baseline characteristics at the index date in unmatched and matched cohorts are summarized for each group and standardized mean differences between groups were calculated. All analyses for endpoints were performed on matched cohorts.

The primary endpoint was evaluated by comparing the percentages of patients receiving prescriptions for antidepressants between the ADHD group and the control group using Fisher's exact test with a significance level of 5\% (twosided). This comparison was performed for each 1-month period throughout the follow-up period. Multiplicity was not adjusted for in this study. The percentage of patients receiving prescriptions for antidepressants was calculated by dividing the number of patients in each group receiving prescriptions for antidepressants, within each month, by the total number of patients in each group.

The mean prescribed dosage of antidepressants per patient per month (in IMP equivalents) was analyzed for each group as one of the secondary endpoints. Two methods were applied: the total monthly prescribed dosage (in IMP equivalents) in each group was divided by the number of patients in the group (by total patients) and divided by the number of patients receiving prescriptions for antidepressants in the relevant month in the group (eg, by patients receiving antidepressants). These mean prescribed antidepressant dosages were compared between the ADHD group and the control group using Student's $t$-test with a significance level of 5\% (two-sided). Comparisons were performed between baseline and each 1-month period of the follow-up. Adjustment for confounding factors, namely prescriptions for anxiolytics or hypnotics, was also performed on this endpoint.

For the other secondary endpoints, the percentages of patients receiving prescriptions for anxiolytics or hypnotics, the same method used for the calculation of the primary endpoint was applied. The population used for the analyses of these secondary endpoints was patients who were included in the population used for the analysis of the primary endpoint and who received a prescription for anxiolytics or hypnotics at the beginning of the baseline period. In the same population, the mean prescribed dosage of anxiolytics per patient per month and that of hypnotics (both in DZP equivalents) were analyzed using the same analysis methods as for the mean prescribed dosage of antidepressants.

As exploratory analyses, the percentage of patients receiving prescriptions for antidepressants and the mean prescribed antidepressant dosage per patient per month (in IMP equivalents) were analyzed using patients' age as a stratification factor ( $<30$ years and $\geq 30$ years of age). An age of 30 years was selected because this was the approximate median age of the patients included in this study (range 20-40 years). The same analysis methods used for the primary and secondary endpoints were used for these analyses. The prescription rates calculated in the present study were not considered to be indicative of patients' adherence to the medication prescribed.

\section{Results}

\section{Patient Disposition and Demographics}

A total of $17,058,880$ patient records were in the JAMDAS database at the time of data extraction on 31 March 2020. The number of patients prescribed both ADHD medication and antidepressants was 7939, and 4414 of these were aged 20-40. In total, 239 patients in the ADHD group and 10,485 patients in the control group met the criteria for inclusion, and records spanning March 2015 to March 2020 were extracted. Of the 10,485 patients in the control group, 239 were included in the matched cohort by propensity score matching (Figure 2).

The patient demographic and baseline characteristics of the unmatched and matched cohorts are shown in Table 1. In the unmatched cohort, the percentage of patients aged $<30$ years was higher in the ADHD group (50.6\%) than in the control group (38.2\%), and the percentage of patients with diagnosed MDD was similar in the ADHD group (96.7\%) and in the control group (90.0\%). The dosage of antidepressants (mean \pm standard deviation) at the index date was also higher in the ADHD group (124.3 \pm $79.4 \mathrm{mg})$ than in the control group $(77.7 \pm 55.5 \mathrm{mg})$. Whereas, in the matched cohort, the percentages of patients aged $<30$ years and of patients with MDD, and the dosage of antidepressants was similar in the ADHD group $(50.6 \%, 96.7 \%$, and $124.3 \pm 79.4 \mathrm{mg}$, respectively) and in the control group $(51.0 \%, 90.8 \%$, and $125.4 \pm$ $80.5 \mathrm{mg}$, respectively). The standardized mean differences of all covariates used for the matching were $<0.1$, showing that the one-to-one matching was appropriately performed.

\section{Primary Endpoint}

The percentages of patients receiving prescriptions for antidepressants in each 1-month period of the follow-up are shown in Figure 3. There were trends towards 


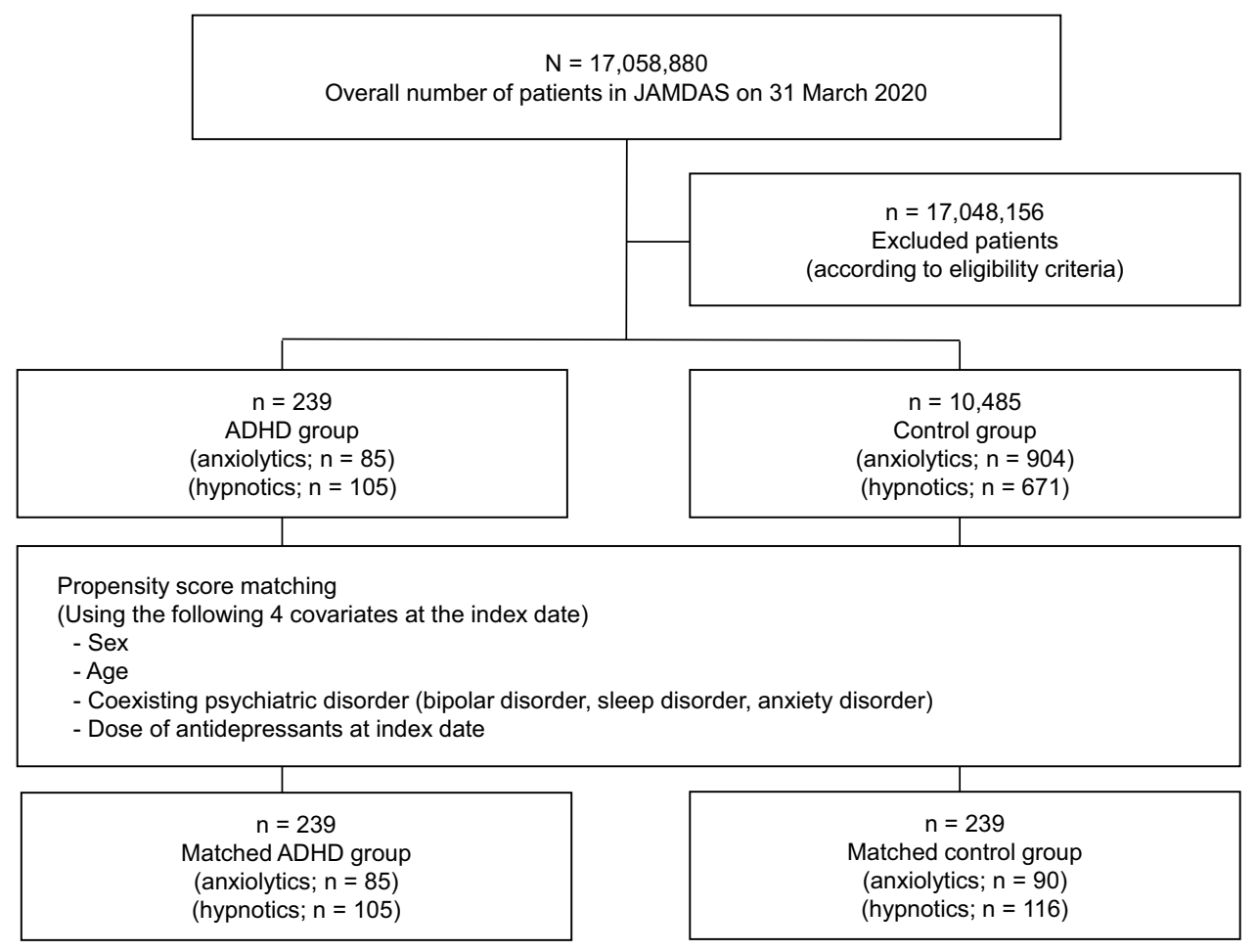

Figure 2 Patient disposition.

Notes: "Anxiolytics" and "hypnotics" in each group refer to patients who received a prescription for anxiolytics and hypnotics, respectively, I month prior to the index date (in the ADHD group) or at the index date (in the control group).

Abbreviations: ADHD, attention-deficit/hyperactivity disorder; JAMDAS, Japanese Medical Data Survey.

decreases in the percentage of patients prescribed antidepressants from baseline in both groups, to $94.1 \%$ in the ADHD group and $89.5 \%$ in the control group during the first 1-month period, and $77.0 \%$ and $78.7 \%$, respectively, during the last 1-month period. No significant difference between the groups was observed in any month of the follow-up.

\section{Secondary Endpoints}

The mean prescribed dosages of antidepressants per patient per month by the number of total patients and patients receiving antidepressants are presented in Figure $4 \mathrm{~A}$ and $\mathrm{B}$, respectively. No significant difference between the groups was observed in the mean prescribed dosage using either of the analysis methods at baseline or in any month of the follow-up. The mean prescribed dosages assessed using total patients were slightly lower than those at baseline in both groups in most months of the follow-up. However, the mean prescribed dosages assessed using patients receiving antidepressants showed slight increases over 6 months in both groups. After adjustment for the potential confounding factors of anxiolytic or hypnotic prescriptions, the results remained non-significant at all time points (data not shown).

The percentages of patients receiving prescriptions for anxiolytics were analyzed using the data of 85 patients in the ADHD group and 90 patients in the control group. Anxiolytic use generally decreased over 6 months in both groups (ADHD group, $-24.7 \%$; control group, $-27.8 \%$; Figure 5). The mean prescribed dosages of anxiolytics per patient per month across all patients and timepoints, including baseline, were generally lower in the ADHD group, ranging from $167.5 \mathrm{mg}$ to $234.0 \mathrm{mg}$ in the ADHD group and from $224.2 \mathrm{mg}$ to $284.9 \mathrm{mg}$ in the control group (Supplementary Table 1). The mean prescribed anxiolytic dosages in patients receiving anxiolytics were also lower in the ADHD group across all time points, including baseline, ranging from $206.3 \mathrm{mg}$ to $255.0 \mathrm{mg}$ compared with $284.9 \mathrm{mg}$ to $311.6 \mathrm{mg}$ in the control group (Supplementary Table 1).

The percentages of patients receiving prescriptions for hypnotics decreased over 6 months in both groups (ADHD group, $-20.0 \%$; control group, $-19.8 \%$; Figure 6 ). The mean prescribed dosages of hypnotics per patient per month across all patients and timepoints, including 


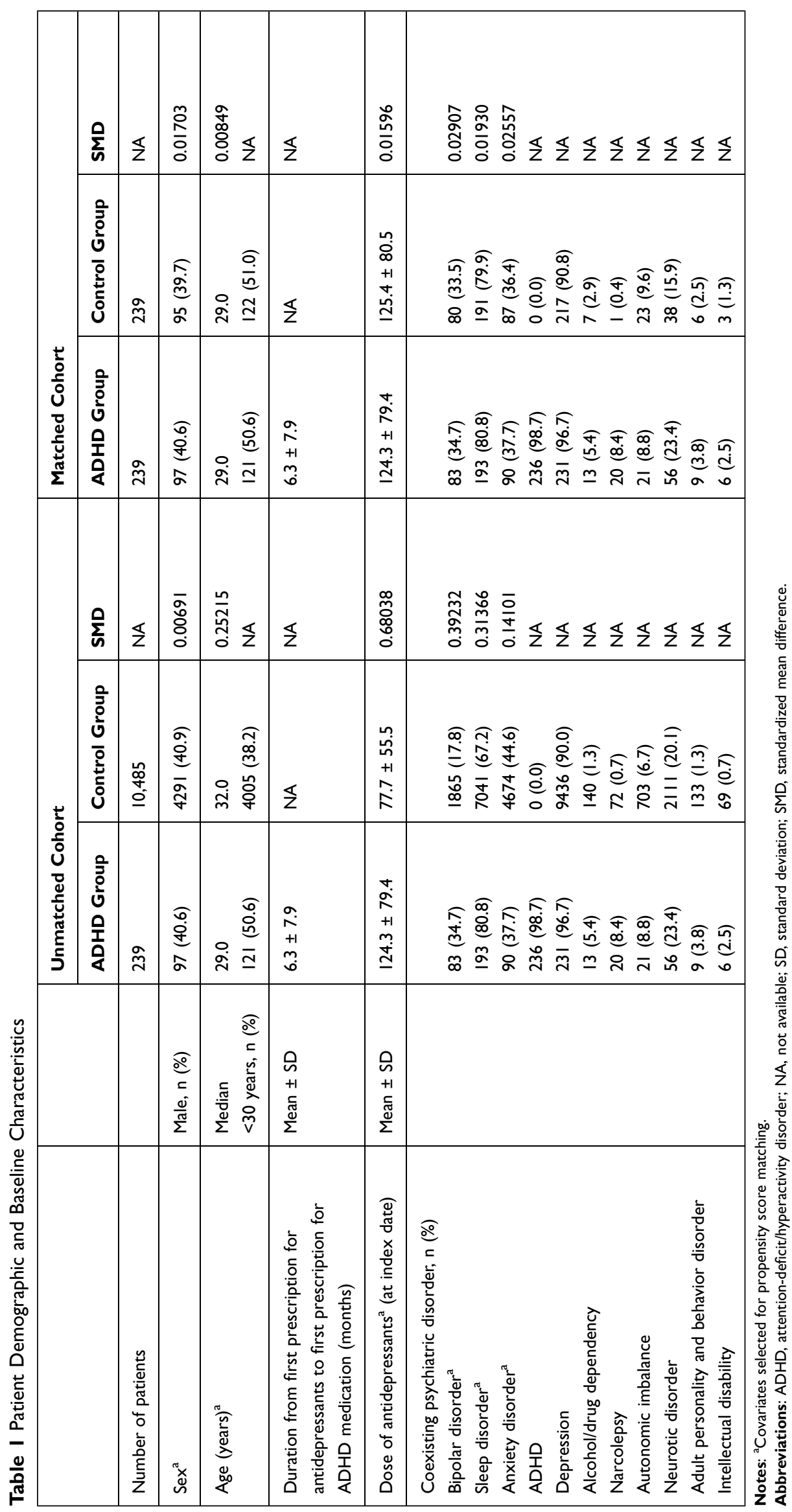




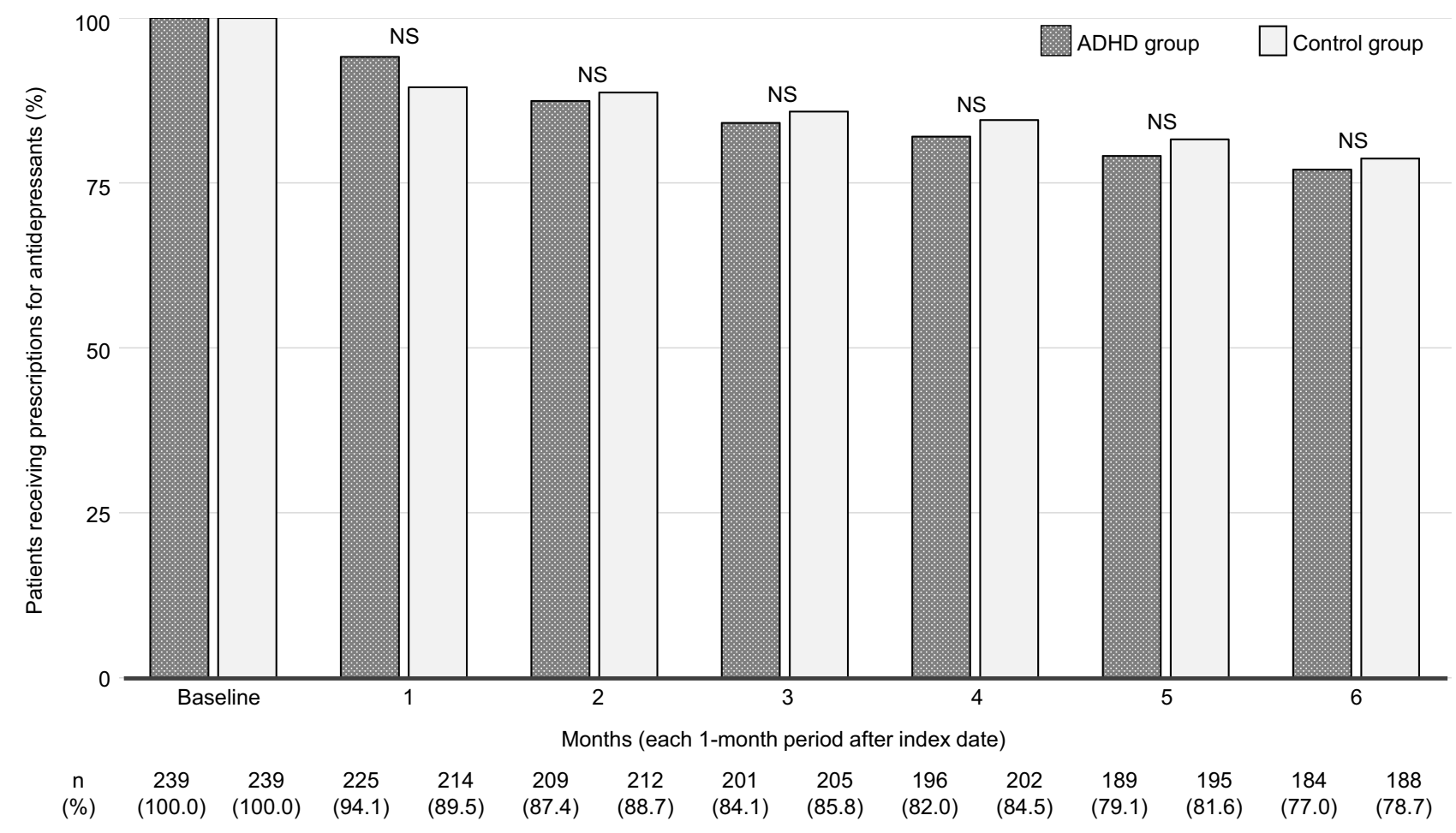

Figure 3 Percentage of patients receiving prescriptions for antidepressants in matched cohort.

Abbreviations: ADHD, attention-deficit/hyperactivity disorder; NS, not significant.

baseline, were generally lower in the ADHD group, ranging from $162.9 \mathrm{mg}$ to $197.9 \mathrm{mg}$, compared with $215.7 \mathrm{mg}$ to $254.6 \mathrm{mg}$ in the control group (Supplementary Table 2). The mean prescribed dosages of hypnotics across all timepoints, including baseline, in patients prescribed hypnotics were also generally lower the ADHD group, ranging from $181.5 \mathrm{mg}$ to $217.7 \mathrm{mg}$, compared with $241.9 \mathrm{mg}$ to $269.1 \mathrm{mg}$ in the control group (Supplementary Table 2).

In addition to the analysis of prescriptions for hypnotics with conversion values to DZP, prescriptions for suvorexant and ramelteon were summarized. In the matched ADHD and the matched control groups, 56 and 36 patients received prescriptions for suvorexant and/or ramelteon through the observational period, respectively.

\section{Exploratory Analyses}

The results of the exploratory analyses were generally consistent with the primary and secondary endpoint results. No obvious difference between subgroups by age ( $<30$ years and $\geq 30$ years of age) was observed, and the number of patients receiving antidepressant prescriptions, and the dosages, were similar between groups (data not shown).

\section{Discussion}

In this study using data from electronic medical records, we examined the effect of add-on ADHD medication on existing prescriptions for antidepressants, anxiolytics, and hypnotics in adults with ADHD. Our data showed that the number of patients receiving antidepressant prescriptions, and the dosages, were similar between patients receiving prescriptions for ADHD medication plus antidepressants and patients receiving prescriptions for antidepressants without being prescribed ADHD medications, of whom most were diagnosed with depression.

A previous study that investigated the prevalence of ADHD symptoms by severity of MDD showed that ADHD symptom rates in patients with MDD were higher in patients with more severe depression. ${ }^{8}$ Another study showed that treatment resistance to antidepressants was greater in patients who had comorbid ADHD than in those with MDD only. ${ }^{13}$ These reports suggest that among patients receiving antidepressants, those with comorbid ADHD tend to have more severe depressive symptoms, and thus, may require higher dosages of MDD medications, than patients without ADHD. Our data revealed that before matching, antidepressant dosage was higher in the patient group prescribed ADHD 
A

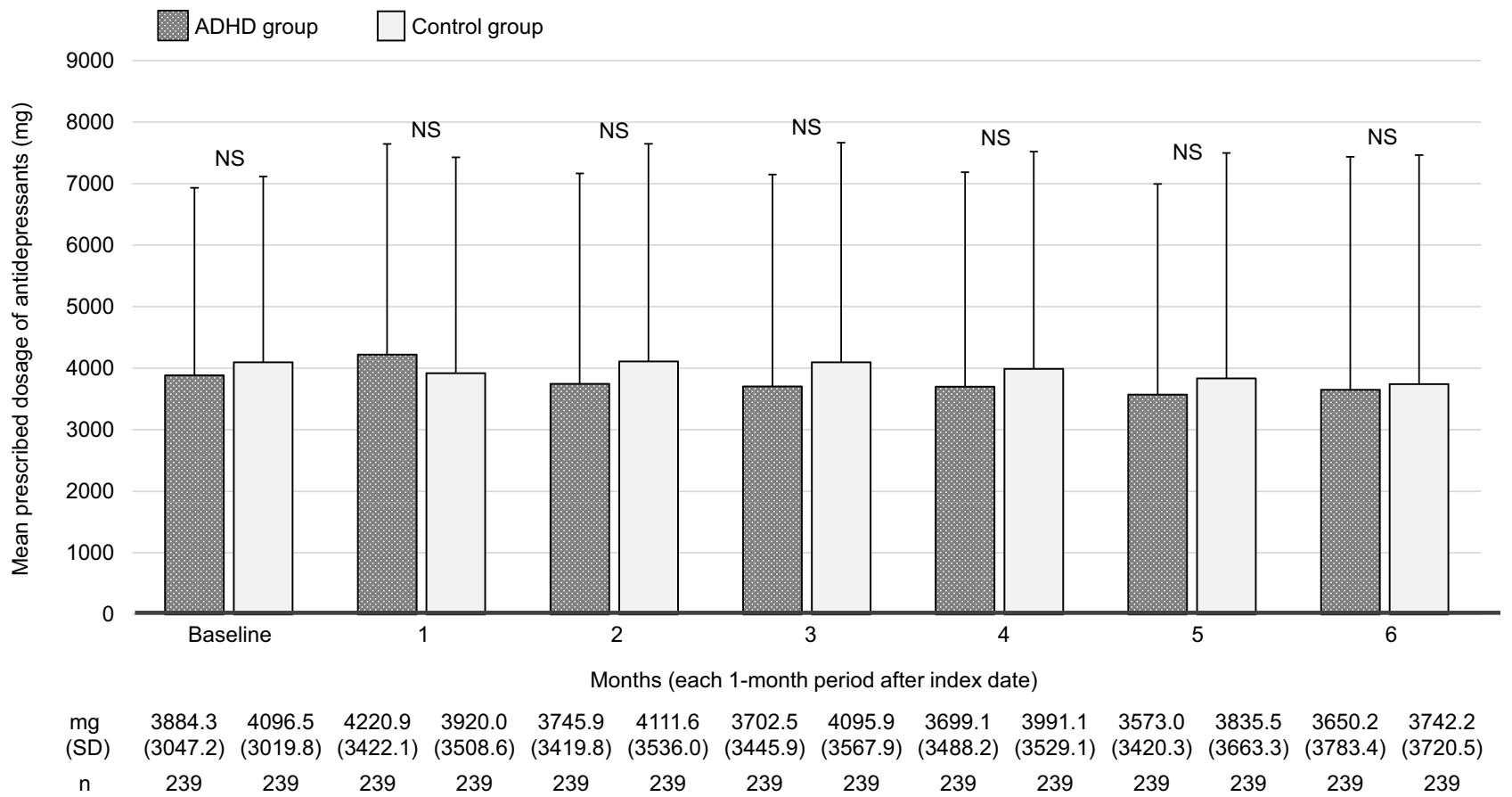

B

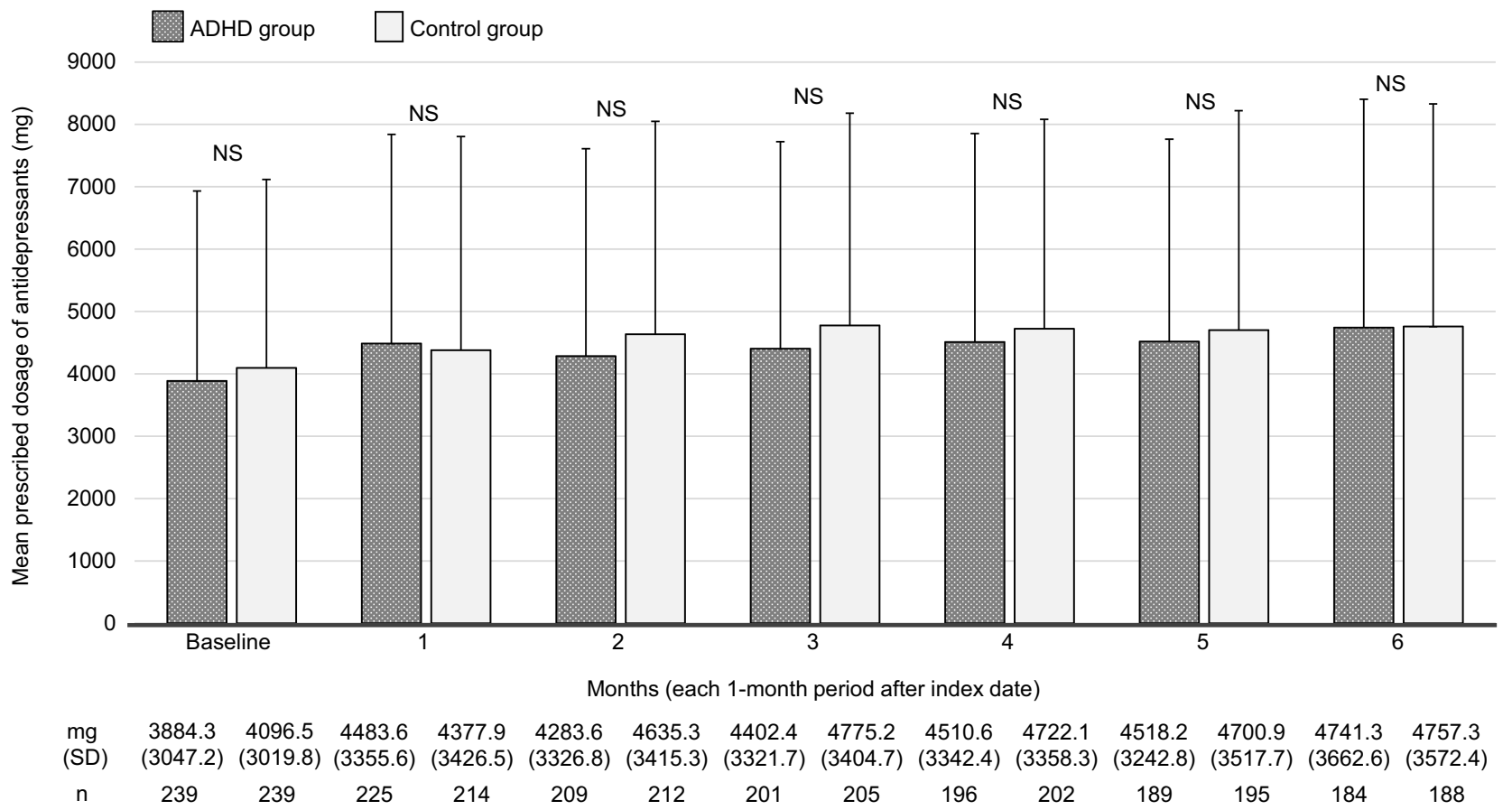

Figure 4 (A) Mean prescribed dosage of antidepressants (in imipramine equivalents) in matched cohort by total patients. (B) Mean prescribed dosage of antidepressants (in imipramine equivalents) in matched cohort by patients receiving antidepressants.

Abbreviations: ADHD, attention-deficit/hyperactivity disorder; NS, not significant; SD, standard deviation. 


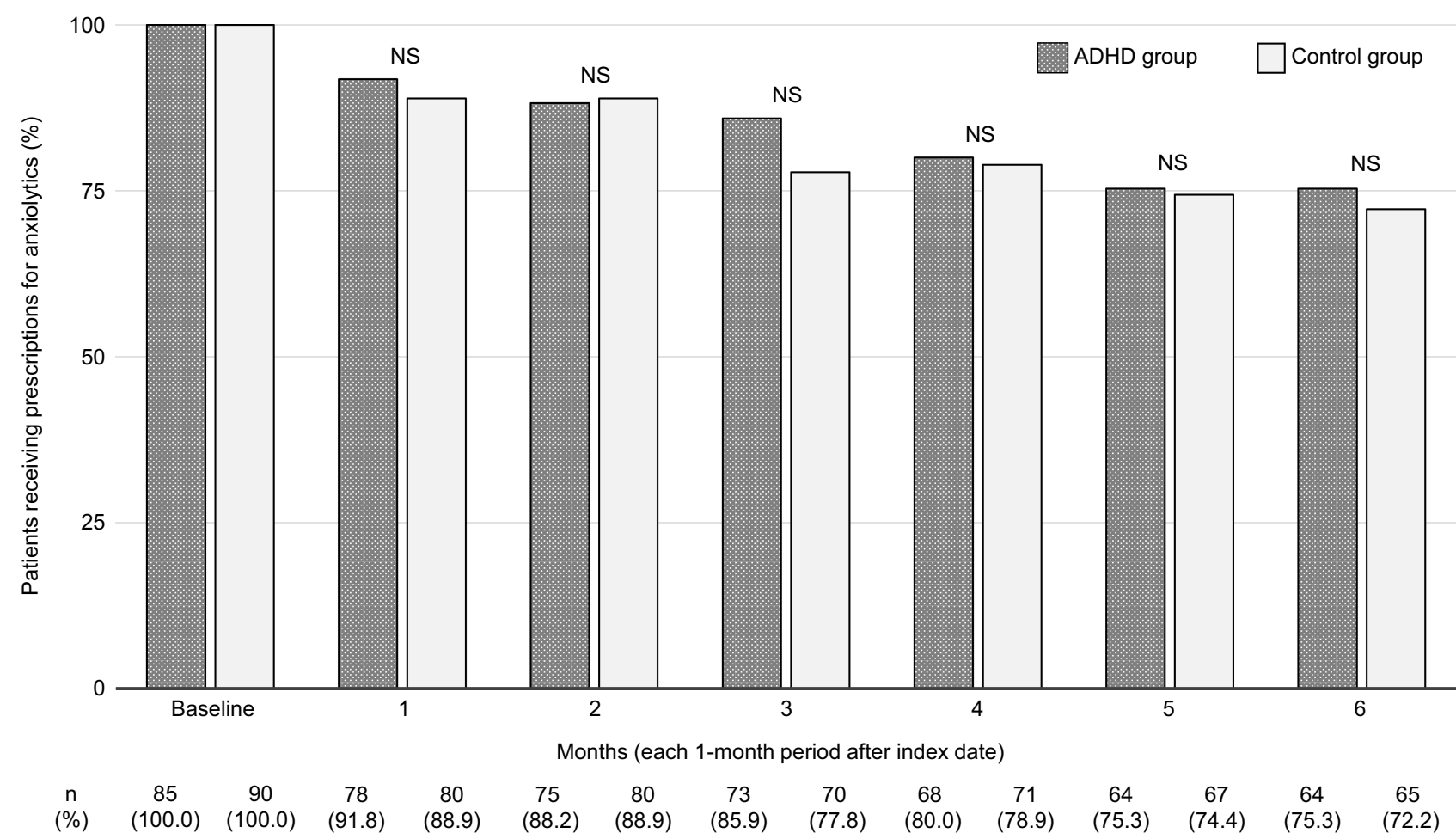

Figure 5 Percentage of patients receiving prescriptions for anxiolytics in matched cohort. Abbreviations: ADHD, attention-deficit/hyperactivity disorder; NS, not significant.

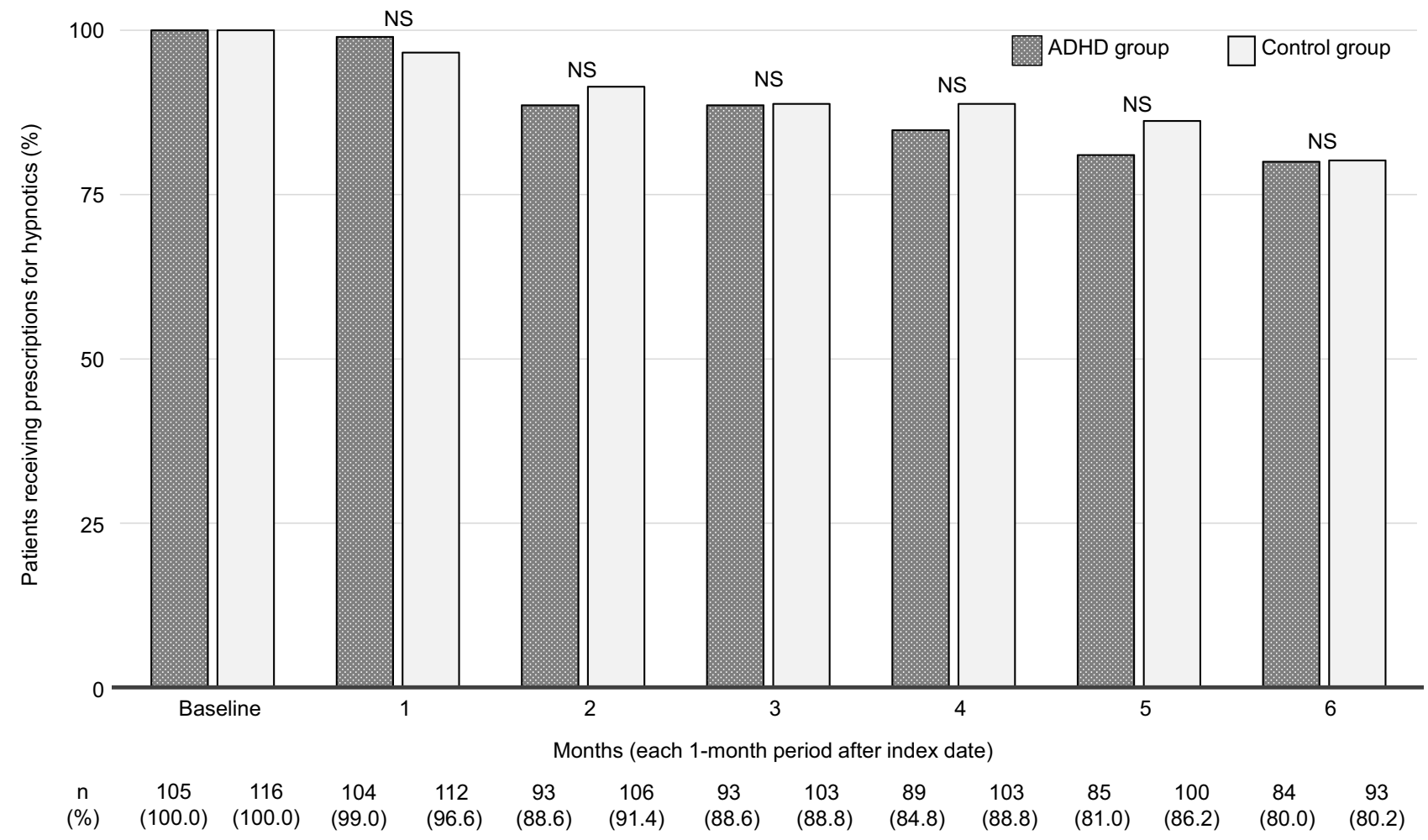

Figure 6 Percentage of patients receiving prescriptions for hypnotics in matched cohort.

Abbreviations: ADHD, attention-deficit/hyperactivity disorder; NS, not significant. 
medication than in the control group. However, our results using the matched population, in which antidepressant dosage was one of the covariates (and therefore the severity of depressive symptoms could be assumed as comparable between the groups), showed that even when ADHD medication was added, antidepressant use was similar between the groups. This suggests that patients receiving prescriptions for ADHD medication generally may not have had worse depressive symptoms than those in the control group for at least 6 months after the prescription for ADHD medication was added, despite patients with ADHD often having more severe depressive symptoms and increased resistance to treatment. ${ }^{8,13}$ Additionally, the prescription of antidepressants remained similar between groups when adjusted for confounding factors such as the prescription of anxiolytics and hypnotics. ADHD treatment may reduce the onset and severity of depressive disorders in the future. ${ }^{14}$

Patients with depressive symptoms often have comorbid symptoms such as anxiety and insomnia, and consequently, anxiolytics and hypnotics are often prescribed in this population. In this study, the prescribed dosages of anxiolytics and hypnotics were generally lower in the ADHD group than in the control group. The prescription of anxiolytics did not show large changes even in the ADHD group, suggesting that patients prescribed ADHD medication do not require higher levels of treatment for anxiety than patients with MDD only. The prescription of hypnotics with DZP conversion values was similar to that of anxiolytics, even in the ADHD group. A previous study reported that ADHD medication effectively improved symptoms of ADHD and comorbid anxiety disorder and it was well tolerated in the adult ADHD patients with social anxiety disorder. ${ }^{15}$ This suggest that physicians may have expected ADHD medications to be effective against anxiety disorder as a comorbidity, and the amount of those prescriptions was low. Moreover, this may reflect physicians prescribing lower doses of medications with dependence liability, such as those in the benzodiazepine class, to patients with ADHD symptoms, in order to decrease the likelihood of dependence on, or abuse of, these medications.

In the case of depression as a secondary disorder of ADHD, we had expected a decrease over time in the percentage of patients receiving prescriptions for antidepressants and the mean prescribed dosage because the reduction in ADHD symptoms by ADHD medication caused an improvement in depression symptoms, but our results did not support this. This could be because the 7-month observation period was too short: from the viewpoint of physicians in clinical practice, at least 1-year of observation after the introduction of a prescription for ADHD medication would be required to detect any changes in antidepressant prescriptions, and longer-term observation may substantiate these hypotheses. ${ }^{14}$ Further investigation including this point is required to understand the prescription status of antidepressants in patients with possible ADHD.

The limitations of our study include the relatively small cohort size; therefore, larger studies are needed. It is not clear whether the patients considered to have depression in this study had a clinical diagnosis of depression or not; rather, the depression status was based on the information provided in the electronic medical charts. Additionally, our assessment of the presence of psychiatric disorders including ADHD, depression, anxiety disorder, and sleep disorder was not based on a clinical diagnosis listed in the patient's medical record but was assumed based on the prescription of medications. Patient symptoms and medication adherence were not assessed, so no correlations with these can be drawn. Prescription rates are not indicative of adherence to treatment. The prescription history of antidepressants prior to our observation may also have varied considerably both between and within groups. The database used in this study only included data from general practitioners and not from hospitals; therefore, the generalization of these results is limited when considering potential bias in the target population. In the ADHD group, the duration of treatment for depression is unknown. Thus, there is also a potential selection bias towards patients who have recently started antidepressants in the control group.

Additionally, the database recognizes the same patient who visits multiple clinics as different individuals, and it is also possible that patients were issued other prescriptions from other hospitals not represented in the database. There may also have been other relevant covariates that were not adjusted for. Finally, the 7-month observation period may have been too short to detect any changes in antidepressant prescriptions, or to detect any reductions in antidepressant dosage, after the start of ADHD treatment. It would be difficult to obtain sufficient data from the JAMDAS database for an observation period longer than this because after 7 months, the number of patients in the database decreases considerably. The prescription issuing frequency should also be considered. 


\section{Conclusion}

The two groups in the present study were medicated in a similar manner with respect to their depressive symptoms over time (6 months). These results suggest that, in patients with depression and comorbid ADHD, depressive symptoms may be adequately controlled following the initiation of ADHD medication without requiring antidepressant dose increases, even though depression is more likely to be more severe in people with ADHD than in those without. Therefore, in patients presenting with severe and persistent depressive symptoms, the coexistence of ADHD should be considered in the earlier stages of treatment.

\section{Data Sharing Statement}

Due to the conditions for use of data extracted from the database, the data used in this study cannot be shared.

\section{Ethics Approval and Informed Consent}

This research was conducted in accordance with the Declaration of Helsinki and the Ethical Guidelines for Medical and Biological Research Involving Human Subjects (Ethical Guidelines) (https://www.lifescience. mext.go.jp/bioethics/seimeikagaku_igaku.html), and permission was obtained from the proprietors of the JAMDAS database. However, because the data used in this research (JAMDAS database) were already anonymized, individuals could not be identified from the data and informed consent from patients included in this study was not required. Therefore, protection for personal information and ethical consideration were not required for this research.

\section{Consent for Publication}

Not applicable. All figures and tables are original.

\section{Acknowledgments}

The authors would like to thank Ms. Manami Yoshida and Mr. Ryo Kiguchi of Shionogi \& Co., Ltd., for their planning of the study, including development of the protocol and statistical analysis plan, and interpretation of the data, and Dr. Nobuhiro Nishigaki of Takeda Pharmaceutical Co., Ltd., for providing an objective review of the interpretation of the data and discussions. We also thank Mr. Atsushi Mizukami and Mr. Masao Shionoya of Mebix Inc. (https://www2.mebix.co.jp/), for providing data analysis and medical writing support, and Ms. Ikuko Tanaka and Dr. Wendy Brooks of Edanz (www.edanz. com), for providing medical writing support, which was funded by Shionogi \& Co., Ltd.

\section{Author Contributions}

All authors made a significant contribution to the work reported, whether that is in the conception, study design, execution, acquisition of data, analysis and interpretation, or in all these areas; took part in drafting, revising or critically reviewing the article; gave final approval of the version to be published; have agreed on the journal to which the article has been submitted; and agree to be accountable for all aspects of the work.

\section{Funding}

This study was funded by Shionogi \& Co., Ltd.

\section{Disclosure}

Chika Sakai was an employee of Shionogi \& Co., Ltd. at the time the study was conducted and holds stock in Shionogi \& Co., Ltd. Chika Sakai is currently affiliated with Janssen Pharmaceutical KK, Tokyo, Japan. Toshinaga Tsuji is a full-time employee of Shionogi \& Co., Ltd. and holds stock in Shionogi \& Co., Ltd. and Takeda Pharmaceutical Co., Ltd. Toru Nakai is a full-time employee of, and holds stock in, Shionogi \& Co., Ltd. Yuki Namba is a full-time employee of, and holds stock in, Shionogi \& Co., Ltd. Hirokazu Mishima is a full-time employee of, and holds stock in, Shionogi \& Co., Ltd. Masakazu Fujiwara was an employee of Shionogi \& Co., Ltd. at the time the study was conducted and holds stock in Shionogi \& Co., Ltd. Hisato Matsunaga has received grants from Shionogi \& Co., Ltd., Otsuka Pharmaceutical Co., Ltd., Eisai Co., Ltd., Dainippon Sumitomo Pharma Co., Ltd., Takeda Pharmaceutical Co., Ltd., and Daiichi Sankyo Company, Limited, and personal fees from Eisai Co., Ltd., MSD K.K., Otsuka Pharmaceutical Co., Ltd., Ono Pharmaceutical Co., Ltd., Janssen Pharmaceutical K. K., Shionogi \& Co., Ltd., Yoshitomiyakuhin Corporation, Dainippon Sumitomo Pharma Co., Ltd., Mitsubishi Tanabe Pharma Corporation, Takeda Pharmaceutical Co., Ltd., and Eli Lilly Japan K.K. The authors report no other conflicts of interest in this work.

\section{References}

1. American Psychiatric Association. Neurodevelopmental disorders: attention-deficit/hyperactivity disorder. In: Diagnostic and Statistical Manual of Mental Disorders. 5th ed. DSM-5. Washington: American Psychiatric Association; 2013. 
2. Willoughby MT. Developmental course of ADHD symptomatology during the transition from childhood to adolescence: a review with recommendations. J Child Psychol Psychiatry. 2003;44(1):88-106. doi:10.1111/1469-7610.t01-1-00104

3. Lara C, Fayyad J, de Graaf R, et al. Childhood predictors of adult attention-deficit/hyperactivity disorder: results from the World Health Organization World Mental Health Survey Initiative. Biol Psychiatry. 2009;65(1):46-54. doi:10.1016/j.biopsych.2008.10.005

4. Biederman J, Petty CR, Clarke A, Lomedico A, Faraone SV. Predictors of persistent ADHD: an 11-year follow-up study. $J$ Psychiatr Res. 2011;45(2):150-155. doi:10.1016/j.jpsychire s.2010.06.009

5. Moffitt TE, Houts R, Asherson P, et al. Is adult ADHD a childhood-onset neurodevelopmental disorder? Evidence from a four-decade longitudinal cohort study. Am J Psychiatry. 2015;172 (10):967-977. doi:10.1176/appi.ajp.2015.14101266

6. Faraone SV, Biederman J. What is the prevalence of adult ADHD? Results of a population screen of 966 adults. J Atten Disord. 2005;9 (2):384-391. doi:10.1177/1087054705281478

7. Katzman MA, Bilkey TS, Chokka PR, Fallu A, Klassen LJ. Adult ADHD and comorbid disorders: clinical implications of a dimensional approach. BMC Psychiatry. 2017;17(1):302. doi:10.1186/s12888-017-1463-3

8. Bron TI, Bijlenga D, Verduijn J, Penninx BW, Beekman AT, Kooij JJ. Prevalence of ADHD symptoms across clinical stages of major depressive disorder. J Affect Disord. 2016;197:29-35. doi:10.1016/j. jad.2016.02.053

9. Wajszilber D, Santiseban JA, Gruber R. Sleep disorders in patients with ADHD: impact and management challenges. Nat Sci Sleep. 2018;10:453-480. doi:10.2147/NSS.S163074
10. Instanes JT, Klungsøyr K, Halmøy A, Fasmer OB, Haavik J. Adult ADHD and comorbid somatic disease: a systematic literature review. $J$ Atten Disord. 2018;22(3):203-228. doi:10.1177/10870547 16669589

11. Mattos P, Louzã MR, Palmini AL, de Oliveira IR, Rocha FL. A multicenter, open-label trial to evaluate the quality of life in adults with ADHD treated with long-acting methylphenidate (OROS MPH): Concerta Quality of Life (CONQoL) study. J Atten Disord. 2013;17 (5):444-448. doi:10.1177/1087054711434772

12. Inada T, Inagaki A. Psychotropic dose equivalence in Japan. Psychiatry Clin Neurosci. 2015;69(8):440-447. doi:10.1111/ pcn. 12275

13. Chen MH, Pan TL, Hsu JW, et al. Attention-deficit hyperactivity disorder comorbidity and antidepressant resistance among patients with major depression: a nationwide longitudinal study. Eur Neuropsychopharmacol. 2016;26(11):1760-1767. doi:10.1016/j. euroneuro.2016.09.369

14. Chang Z, D'Onofrio BM, Quinn PD, et al. Medication for attention-deficit/hyperactivity disorder and risk for depression: a nationwide longitudinal cohort study. Biol Psychiatry. 2016;80 (12):916-922. doi:10.1016/j.biopsych.2016.02.018

15. Adler LA, Liebowitz M, Kronenberger W, et al. Atomoxetine treatment in adults with attention-deficit/hyperactivity disorder and comorbid social anxiety disorder. Depress Anxiety. 2009;26 (3):212-221. doi:10.1002/da.20549
Neuropsychiatric Disease and Treatment

\section{Publish your work in this journal}

Neuropsychiatric Disease and Treatment is an international, peerreviewed journal of clinical therapeutics and pharmacology focusing on concise rapid reporting of clinical or pre-clinical studies on a range of neuropsychiatric and neurological disorders. This journal is indexed on PubMed Central, the 'PsycINFO' database and CAS, and

\section{Dovepress}

is the official journal of The International Neuropsychiatric Association (INA). The manuscript management system is completely online and includes a very quick and fair peer-review system, which is all easy to use. Visit http://www.dovepress.com/testimonials.php to read real quotes from published authors. 